\title{
Approximation for $q$-Chlodowsky Operators via Statistical Convergence with Respect to Power Series Method *
}

\author{
Halime Taşer* and Tuğba Yurdakadim
}

\begin{abstract}
Many results which are obtained or unable to obtained by classical calculus have also been studied by $q$-calculus. It is effective to use $q$-calculus since it acts as a bridge between mathematics and physics. The $q$-analog of Chlodowsky operators has been introduced and the approximation properties of these operators have been studied in [12]. Then in [23], the $q$-analog of Stancu-Chlodowsky operators has been introduced and some approximation results of these operators have been studied via $A$-statistical convergence which is a more general setting. In this paper, we present the approximation properties of $q$-Chlodowsky operators via statistical convergence with respect to power series method. It is noteworthy to mention that statistical convergence and statistical convergence with respect to power series method are incompatible.
\end{abstract}

Keywords: $q$-calculus; Chlodowsky operators; approximation theory; power series method; statistical convergence.

AMS Subject Classification (2020): Primary: 40G10; Secondary: 41A36.

*Corresponding Author

\section{Introduction and Preliminaries}

In approximation theory, Bernstein operators have different applications. With the use of these operators, it is possible to give an understandable and easy proof of Weierstrass's theorem. This is the most important application of these operators. The classical Bernstein operators have been introduced and discussed in detailed in $[1,2,5,13,15,25]$. Since $q$-calculus acts as a bridge between mathematics and physics, the $q$-analog of Bernstein operators have been introduced by Lupaş [16]. Different type of $q$-Bernstein operators has also been introduced by Phillips [21] and Ostrovska [19] have investigated the approximation properties of these operators. Karsli and Gupta [12] have introduced $q$-Chlodowsky operators which extend $q$-Bernstein operators to an unbounded interval.

\footnotetext{
*This study has been produced by the master thesis titled with "Approximation for $q$-Chlodowsky Operators via Statistical Convergence With Respect To Power Series Method", conducted under the supervision of Assoc. Prof. Dr. Tuğba Yurdakadim at Bilecik Şeyh Edebali University, Institute of Graduate Studies, Department of Mathematics, Bilecik, Turkey.
} 
The important results in approximation theory have also been studied by using different concepts of convergences such as statistical convergence, ideal convergence, summation process $[3,4,8,11,20,24]$. It is effective to use these concepts since they make a nonconvergent sequence to converge.

In the present paper, we study the approximation properties of $q$-Chlodowsky operators via statistical convergence with respect to power series method. In [26], such examples have been provided to show that statistical convergence and statistical convergence with respect to power series method do not imply each other.

This paper is organized as follows:

The first section is devoted to basic definitions, notations and also well known results. The second section is devoted to our main results and in the third section we will give an application.

Now, let us recall basic definitions, notations and also the well known results which we need throughout the paper.

The density of the subset $E \subseteq \mathbb{N}_{0}$ is given by

$$
\delta(E):=\lim _{n \rightarrow \infty} \frac{1}{n+1}|\{j \leqslant n: j \in E\}|
$$

whenever the limit exists where the vertical bars indicate the cardinality of enclosed set and $\mathbb{N}_{0}$ is the set of all nonnegative integers. A sequence $x=\left(x_{j}\right)$ is called statistically convergent to $L[9,10,22]$ if for every $\varepsilon>0$,

$$
\lim _{n \rightarrow \infty} \frac{1}{n+1}\left|\left\{j \leqslant n:\left|x_{j}-L\right| \geqslant \varepsilon\right\}\right|=0
$$

that is, $\delta\left(E_{\varepsilon}\right)=0$ for any $\varepsilon>0$ where $E_{\varepsilon}=\left\{j \in \mathbb{N}_{0}:\left|x_{j}-L\right| \geqslant \varepsilon\right\}$.

By assuming that $\left(p_{j}\right)$ is nonnegative real sequence such that $p_{0}>0$ and the corresponding power series $p(t):=\sum_{j=0}^{\infty} p_{j} t^{j}$ has radius of convergence $R$ with $0<R \leqslant \infty$. Now the definition of power series method is as follows :

Let

$$
C_{p}:=\left\{f:(-R, R) \rightarrow \mathbb{R} \mid \lim _{0<t \rightarrow R^{-}} \frac{1}{p(t)} f(t) \quad \text { exists }\right\}
$$

and

$$
C_{P_{p}}:=\left\{x=\left(x_{k}\right) \mid p_{x}(t):=\sum_{j=0}^{\infty} p_{j} t^{j} x_{j} \quad \text { has radius of convergence } \geqslant R \quad \text { and } \quad p_{x} \in C_{p}\right\} .
$$

The functional $P_{p}-\lim : C_{P_{p}} \rightarrow \mathbb{R}$ (for short $P_{p}$ ) defined by

$$
P_{p}-\lim x=\lim _{0<t \rightarrow R^{-}} \frac{1}{p(t)} \sum_{j=0}^{\infty} p_{j} t^{j} x_{j}
$$

is called a power series method and $x$ is said to be $P_{p}$-convergent [6], [14].

A power series method $P_{p}$ is said to be regular if $P_{p}-\lim x=L$ provided that $\lim x=L$ [6].

By combining these concepts, Ünver and Orhan [26] have recently introduced $P_{p}$-statistical convergence and have proved a Korovkin type theorem for a sequence of positive linear operators defined on $C[0,1]$, the space of all continuous functions on the interval $[0,1]$.

Now let us recall the statistical convergence with respect to power series method, i.e., $P_{p}$-statistical convergence. Let $P_{p}$ be a regular power series method and $E \subseteq \mathbb{N}_{0}$. If the limit

$$
\delta_{P_{p}}(E):=\lim _{0<t \rightarrow R^{-}} \frac{1}{p(t)} \sum_{j \in E} p_{j} t^{j}
$$

exists then $\delta_{P_{p}}(E)$ is called the $P_{p}$-density of $E$. Notice that by the definition of a power series method and $P_{p^{-}}$ density it is obvious that $0 \leqslant \delta_{P_{p}}(E) \leqslant 1$ whenever it exists [26].

Let $x=\left(x_{j}\right)$ be a real sequence and let $P_{p}$ be a regular power series method. Then $x$ is said to be $P_{p}$-statistically convergent to $L$ if for any $\varepsilon>0$

$$
\lim _{0<t \rightarrow R^{-}} \frac{1}{p(t)} \sum_{j \in E_{\varepsilon}} p_{j} t^{j}=0
$$


that is, $\delta_{P_{p}}\left(E_{\varepsilon}\right)=0$ for any $\varepsilon>0$. In the case we write $s t_{P_{p}}-\lim x=L[26]$.

Before recalling the $q$-Chlodowsky operators, it is useful to mention certain properties of $q$-calculus. For any fixed real number $q>0$ and nonnegative integer $r$, the $q$-integer of the number $r$ is defined by

$$
[r]_{q}= \begin{cases}\left(1-q^{r}\right) /(1-q), & q \neq 1 \\ r, & q=1\end{cases}
$$

The $q$-factorial is defined by

$$
[r]_{q} != \begin{cases}{[r]_{q}[r-1]_{q} \cdots[1]_{q},} & r=1,2, \ldots \\ 1, & r=0\end{cases}
$$

and $q$-binomial coefficient can be defined as

$$
\left[\begin{array}{l}
k \\
r
\end{array}\right]_{q}=\frac{[k]_{q} !}{[r]_{q} ![k-r]_{q} !}
$$

for integers $k \geqslant r \geqslant 0$ and $q$-binomial coefficients satisfy the following properties:

$$
\left[\begin{array}{c}
k+1 \\
r
\end{array}\right]_{q}=q^{k-r+1}\left[\begin{array}{c}
k \\
r-1
\end{array}\right]_{q}+\left[\begin{array}{l}
k \\
r
\end{array}\right]_{q}
$$

and

$$
\left[\begin{array}{c}
k+1 \\
r
\end{array}\right]_{q}=\left[\begin{array}{c}
k \\
r-1
\end{array}\right]_{q}+q^{r}\left[\begin{array}{l}
k \\
r
\end{array}\right]_{q}
$$

The $q$-analog of $(1-a)^{n}$ is the polynomial

$$
(1-a)_{q}^{n}= \begin{cases}1, & n=0 \\ \prod_{s=0}^{n-1}\left(1-q^{s} a\right), & n \geqslant 1 .\end{cases}
$$

The Bernstein-Chlodowsky operators were defined by Chlodowsky on an unbounded set in 1937 [7] as follows:

$$
C_{n}(f ; x)=\sum_{k=0}^{n} f\left(\frac{k}{n} b_{n}\right)\left[\begin{array}{l}
n \\
k
\end{array}\right]\left(\frac{x}{b_{n}}\right)^{k}\left(1-\frac{x}{b_{n}}\right)^{n-k}
$$

where $0 \leq x \leq b_{n},\left(b_{n}\right)$ is a positive increasing sequence with the properties that, $\lim _{n \rightarrow \infty} b_{n}=\infty$ and $\lim _{n \rightarrow \infty} \frac{b_{n}}{n}=0$. The $q$-Bernstein operators have also been defined by [21] and have been studied by many researchers (see e.g. $[17,18,27]$ etc.)

$$
B_{n, q}(f ; x)=\sum_{k=0}^{n} f\left(\frac{[k]_{q}}{[n]_{q}}\right)\left[\begin{array}{l}
n \\
k
\end{array}\right]_{q} x^{k} \prod_{s=0}^{n-k-1}\left(1-q^{s} x\right) .
$$

With the same motivation in the classical procedure, the $q$-Chlodowsky operators have been defined as:

$$
C_{n, q}(f ; x)=\sum_{k=0}^{n} f\left(\frac{[k]_{q}}{[n]_{q}} b_{n}\right)\left[\begin{array}{l}
n \\
k
\end{array}\right]_{q}\left(\frac{x}{b_{n}}\right)^{k} \prod_{s=0}^{n-k-1}\left(1-q^{s} \frac{x}{b_{n}}\right)
$$

where $0 \leq x \leq b_{n},\left(b_{n}\right)$ is a positive increasing sequence with the property that $\lim _{n \rightarrow \infty} b_{n}=\infty$. In [12] the following theorem has been obtained for $q$-Chlodowsky operators.

Theorem 1. $C_{n, q}$ operators satisfy the following equalities

$$
\begin{aligned}
C_{n, q}(1 ; x) & =1, \\
C_{n, q}(t ; x) & =x, \\
C_{n, q}\left(t^{2} ; x\right) & =x^{2}+\frac{x\left(b_{n}-x\right)}{[n]_{q}} .
\end{aligned}
$$


From Theorem 1 and by direct computations, we have the following equalities:

$$
C_{n, q}\left((t-x)^{2} ; x\right)=\frac{x\left(b_{n}-x\right)}{[n]_{q}}, \quad C_{n, q}((t-x) ; x)=0 .
$$

One can easily observe that $[n]_{q} \rightarrow \frac{1}{1-q}$ as $n \rightarrow \infty$ for $0<q<1$ and it implies that $C_{n, q}\left(t^{2} ; x\right)$ and $C_{n, q}\left((t-x)^{2} ; x\right)$ do not converge to $x^{2}$ and 0 respectively, as $n \rightarrow \infty$.

In order to overcome this difficulty, we replace $q$ by $\left(q_{n}\right)$ where $\left(q_{n}\right)$ is a sequence of real numbers such that $0<q_{n}<1$,

$$
s t_{P_{p}}-\lim q_{n}=1
$$

and

$$
s t_{P_{p}}-\lim \frac{b_{n}}{[n]_{q_{n}}}=0 .
$$

Now let us recall the modulus of continuity of $f, \omega(f, \delta)$ is defined by

$$
\omega(f, \delta)=\sup _{\substack{|x-y| \leq \delta \\ x, y \in[0, B]}}|f(x)-f(y)| .
$$

It is well known that for a function $f \in C[0, B]$,

$$
\lim _{\delta \rightarrow 0^{+}} \omega(f, \delta)=0,
$$

and for any $\lambda>0$

$$
\omega(f, \lambda \delta) \leq(1+\lambda) \omega(f, \delta)
$$

\section{Main Results}

In this section, we present our main results which fill the gaps in the existing literature. First of all, we recall the following theorem which states the necessary and sufficient condition for the convergence of a sequence of positive linear operators on $C[0,1]$.

Theorem 2. [26] Let $P_{p}$ be a regular power series method and let $\left(L_{n}\right)$ be a sequence of positive linear operators on $C[a, b]$ such that for $e_{i}(x)=x^{i}, i=0,1,2$

$$
s t_{P_{p}}-\lim \left\|L_{n} e_{i}-e_{i}\right\|=0
$$

then for any $f \in C[a, b]$, we have

$$
s t_{P_{p}}-\lim \left\|L_{n} f-f\right\|=0 .
$$

Under the light of Theorem 2, we are ready to present and prove the following:

Theorem 3. Let $\left(q_{n}\right)$ be a sequence of real numbers such that $0<q_{n}<1, s t_{P_{p}}-\lim q_{n}=1$. Then for any $f \in C[0, \infty)$, we have

$$
s t_{P_{p}}-\lim \left\|C_{n, q_{n}}(f)-f\right\|_{C[0, B]}=0
$$

where $B$ is positive real number.

Proof. Using Theorem 1 and Theorem 2 and since $s t_{P_{p}}-\lim q_{n}=1$ and $s t_{P_{p}}-\lim \frac{b_{n}}{[n]_{q_{n}}}=0$, we obtain the desired result. This completes the proof.

Theorem 4. Let $\left(q_{n}\right)$ be a sequence of real numbers such that $0<q_{n}<1$ and $\operatorname{st}_{P_{p}}-\lim q_{n}=1$. If $f \in C[0, \infty)$, then we have

$$
\left|C_{n, q_{n}}(f ; x)-f(x)\right| \leq 2 \omega\left(f, \sqrt{\frac{x\left(b_{n}-x\right)}{[n]_{q_{n}}}}\right) .
$$


Proof. By using simple calculations, we have

$$
\begin{aligned}
& \left|C_{n, q_{n}}(f ; x)-f(x)\right|=\left|\sum_{k=0}^{n} f\left(\frac{[k]_{q_{n}}}{[n]_{q_{n}}} b_{n}\right)\left[\begin{array}{l}
n \\
k
\end{array}\right]_{q_{n}}\left(\frac{x}{b_{n}}\right)^{k}\left(1-\frac{x}{b_{n}}\right)_{q_{n}}^{n-k}-f(x)\right| \\
& =\left|\sum_{k=0}^{n}\left[f\left(\frac{[k]_{q_{n}}}{[n]_{q_{n}}} b_{n}\right)-f(x)\right]\left[\begin{array}{l}
n \\
k
\end{array}\right]_{q_{n}}\left(\frac{x}{b_{n}}\right)^{k}\left(1-\frac{x}{b_{n}}\right)_{q_{n}}^{n-k}\right| \\
& \leq \sum_{k=0}^{n}\left|f\left(\frac{[k]_{q_{n}}}{[n]_{q_{n}}} b_{n}\right)-f(x)\right|\left[\begin{array}{l}
n \\
k
\end{array}\right]_{q_{n}}\left(\frac{x}{b_{n}}\right)^{k}\left(1-\frac{x}{b_{n}}\right)_{q_{n}}^{n-k} \\
& \leq \sum_{k=0}^{n} \omega\left(f,\left|\frac{[k]_{q_{n}}}{[n]_{q_{n}}} b_{n}-x\right|\right)\left[\begin{array}{l}
n \\
k
\end{array}\right]_{q_{n}}\left(\frac{x}{b_{n}}\right)^{k}\left(1-\frac{x}{b_{n}}\right)_{q_{n}}^{n-k} \\
& =\sum_{k=0}^{n} \omega\left(f, \frac{\delta \mid\left[\frac{\left[k q_{q_{n}}\right.}{[n]_{q_{n}}} b_{n}-x \mid\right.}{\delta}\right)\left[\begin{array}{l}
n \\
k
\end{array}\right]_{q_{n}}\left(\frac{x}{b_{n}}\right)^{k}\left(1-\frac{x}{b_{n}}\right)_{q_{n}}^{n-k}
\end{aligned}
$$

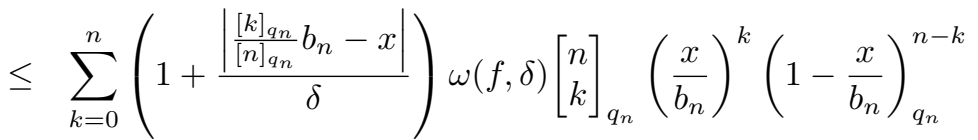

$$
\begin{aligned}
& =\sum_{k=0}^{n} \omega(f, \delta)\left[\begin{array}{l}
n \\
k
\end{array}\right]_{q_{n}}\left(\frac{x}{b_{n}}\right)^{k}\left(1-\frac{x}{b_{n}}\right)_{q_{n}}^{n-k} \\
& +\sum_{k=0}^{n} \frac{\omega(f, \delta)}{\delta}\left|\frac{[k]_{q_{n}}}{[n]_{q_{n}}} b_{n}-x\right|\left[\begin{array}{l}
n \\
k
\end{array}\right]_{q_{n}}\left(\frac{x}{b_{n}}\right)^{k}\left(1-\frac{x}{b_{n}}\right)_{q_{n}}^{n-k} \\
& =\omega(f, \delta) \sum_{k=0}^{n}\left[\begin{array}{l}
n \\
k
\end{array}\right]_{q_{n}}\left(\frac{x}{b_{n}}\right)^{k}\left(1-\frac{x}{b_{n}}\right)_{q_{n}}^{n-k} \\
& +\frac{\omega(f, \delta)}{\delta} \sum_{k=0}^{n}\left|\frac{[k]_{q_{n}}}{[n]_{q_{n}}} b_{n}-x\right|\left[\begin{array}{l}
n \\
k
\end{array}\right]_{q_{n}}\left(\frac{x}{b_{n}}\right)^{k}\left(1-\frac{x}{b_{n}}\right)_{q_{n}}^{n-k} .
\end{aligned}
$$

It is also well known that

$$
\sum_{k=0}^{n}\left[\begin{array}{l}
n \\
k
\end{array}\right]_{q_{n}}\left(\frac{x}{b_{n}}\right)^{k}\left(1-\frac{x}{b_{n}}\right)_{q_{n}}^{n-k}=\left[\frac{x}{b_{n}}+\left(1-\frac{x}{b_{n}}\right)\right]_{q_{n}}^{n}=1
$$

and by using Cauchy-Schwarz inequality, we have

$$
\begin{aligned}
& \sum_{k=0}^{n}\left|\frac{[k]_{q_{n}}}{[n]_{q_{n}}} b_{n}-x\right|\left[\begin{array}{l}
n \\
k
\end{array}\right]_{q_{n}}\left(\frac{x}{b_{n}}\right)^{k}\left(1-\frac{x}{b_{n}}\right)_{q_{n}}^{n-k} \\
& \leq \sum_{k=0}^{n}\left|\frac{[k]_{q_{n}}}{[n]_{q_{n}}} b_{n}-x\right|\left\{\left[\begin{array}{l}
n \\
k
\end{array}\right]_{q_{n}}\left(\frac{x}{b_{n}}\right)^{k}\left(1-\frac{x}{b_{n}}\right)_{q_{n}}^{n-k}\right\}^{1 / 2}\left\{\left[\begin{array}{l}
n \\
k
\end{array}\right]_{q_{n}}\left(\frac{x}{b_{n}}\right)^{k}\left(1-\frac{x}{b_{n}}\right)_{q_{n}}^{n-k}\right\}^{1 / 2} \\
& \leq\left\{\sum_{k=0}^{n}\left(\frac{[k]_{q_{n}}}{[n]_{q_{n}}} b_{n}-x\right)^{2}\left[\begin{array}{l}
n \\
k
\end{array}\right]_{q_{n}}\left(\frac{x}{b_{n}}\right)^{k}\left(1-\frac{x}{b_{n}}\right)_{q_{n}}^{n-k}\right\}^{1 / 2}\left\{\sum_{k=0}^{n}\left[\begin{array}{l}
n \\
k
\end{array}\right]_{q_{n}}\left(\frac{x}{b_{n}}\right)^{k}\left(1-\frac{x}{b_{n}}\right)_{q_{n}}^{n-k}\right\}^{1 / 2} \\
& =\left\{\frac{x\left(b_{n}-x\right)}{[n]_{q_{n}}}\right\}^{1 / 2} .
\end{aligned}
$$

Then

$$
\left|C_{n, q_{n}}(f ; x)-f(x)\right| \leq \omega(f, \delta)+\frac{\omega(f, \delta)}{\delta}\left\{\frac{x\left(b_{n}-x\right)}{[n]_{q_{n}}}\right\}^{1 / 2}
$$


holds. By taking $\delta=\left\{\frac{x\left(b_{n}-x\right)}{[n]_{q_{n}}}\right\}^{1 / 2}$, we have

$$
\left|C_{n, q_{n}}(f ; x)-f(x)\right| \leq 2 \omega(f, \delta)=2 \omega\left(f, \sqrt{\frac{x\left(b_{n}-x\right)}{[n]_{q_{n}}}}\right) .
$$

This completes the proof.

Theorem 5. Let $\left(q_{n}\right)$ be a sequence of real numbers such that $0<q_{n}<1$ and $s t_{P_{p}}-\lim q_{n}=1$. If $f \in C\left[0, b_{n}\right]$, then we have

$$
\left|C_{n, q_{n}}\left(f ; x_{0}\right)-f\left(x_{0}\right)\right| \leq 2 \omega\left(f, \sqrt{\frac{x_{0} b_{n}}{[n]_{q_{n}}}}\right)
$$

where $x_{0} \in\left[0, b_{n}\right]$ and $x_{0}$ is a fixed point.

Proof. The validity of the following is obvious:

$$
\frac{x\left(b_{n}-x\right)}{[n]_{q_{n}}} \leq \frac{x_{0} b_{n}}{[n]_{q_{n}}}
$$

for any fixed point $x_{0}$. One can obtain the remaining part in a similar way in [12]. Therefore we omit the details here.

Theorem 6. Let $\left(q_{n}\right)$ be a sequence of real numbers such that $0<q_{n}<1$ and $\operatorname{st}_{P_{p}}-\lim q_{n}=1$. If $f \in C[0, \infty)$, then we have, for sufficiently large $n$

$$
\left\|C_{n, q_{n}}(f)-f\right\|_{C\left[0, b_{n}\right]} \leq 2 \omega\left(f, \sqrt{\frac{B b_{n}}{[n]_{q_{n}}}}\right)
$$

where $B>0$ is a constant being appeared in Theorem 3.

In [23], the $q$-analog of the Stancu type Bernstein-Chlodowsky operators have been introduced as follows:

$$
C_{n, q}^{\alpha, \beta}(f ; x)=\sum_{k=0}^{n} f\left(\frac{[k]_{q}+[\alpha]_{q}}{[n]_{q}+[\beta]_{q}} b_{n}\right)\left[\begin{array}{l}
n \\
k
\end{array}\right]_{q}\left(\frac{x}{b_{n}}\right)^{k}\left(1-\frac{x}{b_{n}}\right)^{n-k}
$$

where $0 \leq x \leq b_{n},\left(b_{n}\right)$ is a positive increasing sequence with the property that $\lim _{n \rightarrow \infty} b_{n}=\infty$ and $\alpha, \beta$ are positive integers such that $0 \leqslant \alpha \leqslant \beta$.

Observe that when we take $\alpha=\beta=0, q$-analog of the Stancu type Bernstein-Chlodowsky operators coincide with $q$-Bernstein-Chlodowsky operators.

In [23] the following theorem has been obtained for the Stancu type Bernstein-Chlodowsky operators.

Theorem 7. The followings are satisfied for $C_{n, q}^{\alpha, \beta}$;

$$
\begin{aligned}
C_{n, q}^{\alpha, \beta}(1 ; x) & =1, \\
C_{n, q}^{\alpha, \beta}(t ; x) & =\frac{[n]_{q}}{[n]_{q}+[\beta]_{q}} x+\frac{[\alpha]_{q}}{[n]_{q}+[\beta]_{q}} b_{n}, \\
C_{n, q}^{\alpha, \beta}\left(t^{2} ; x\right) & =\frac{[n]_{q}{ }^{2}}{\left([n]_{q}+[\beta]_{q}\right)^{2}}\left(x^{2}+\frac{x\left(b_{n}-x\right)}{[n]_{q}}\right)+\frac{2[\alpha]_{q}[\beta]_{q}}{\left([n]_{q}+[\beta]_{q}\right)^{2}}+\frac{[n]_{q}{ }^{2}}{\left([n]_{q}+[\beta]_{q}\right)^{2}} b_{n}{ }^{2} .
\end{aligned}
$$

Since

$$
s t_{P_{p}}-\lim \frac{b_{n}}{[n]_{q_{n}}}=0,0<q_{n}<1 \text { and } s t_{P_{p}}-\lim q_{n}=1,
$$

we can also obtain analogous results those given in [23] in a similar manner for the concept of $P_{p}$-statistical convergence. 
Recall that for $f \in C[a, b]$ and $t>0$, the Peetre- $K$ Functional is defined by

$$
K(f, \delta):=\inf _{g \in C^{2}[a, b]}\left\{\|f-g\|_{C[a, b]}+t\|g\|_{C^{2}[a, b]}\right\}
$$

where $C^{2}[a, b]=\left\{f \in C[a, b]: f^{\prime}, f^{\prime \prime} \in C[a, b]\right\}$, with the norm

$$
\|g\|_{C^{2}[a, b]}:=\|g\|_{C[a, b]}+\left\|g^{\prime}\right\|_{C[a, b]}+\left\|g^{\prime \prime}\right\|_{C[a, b]} .
$$

It is obtained in [12] that for $g \in C^{2}\left[0, b_{n}\right]$, then we have

$$
\left|C_{n, q}(g ; x)-g(x)\right| \leqslant \frac{x\left(b_{n}-x\right)}{2[n]_{q}}\|g\|_{C^{2}\left[0, b_{n}\right]} .
$$

Theorem 8. Let $\left(q_{n}\right)$ be a sequence of real numbers such that $0<q_{n}<1$ and

$$
s t_{P_{p}}-\lim q_{n}=1 .
$$

If $f \in C[0, \infty)$ and $B>0$ is a constant, then we have

$$
\left\|C_{n, q_{n}}(f)-f\right\|_{C\left[0, b_{n}\right]} \leqslant 2 K\left(f, \frac{B b_{n}}{2[n]_{q_{n}}}\right) .
$$

Proof. From [12], it is known that

$$
\left|C_{n, q_{n}}(f ; x)-f(x)\right| \leqslant\|f-g\|_{C\left[0, b_{n}\right]}\left|C_{n, q_{n}}(1 ; x)\right|+\|f-g\|_{C\left[0, b_{n}\right]}+\left|C_{n, q_{n}}(g ; x)-g(x)\right|
$$

and

$$
\left|C_{n, q_{n}}(f ; x)-f(x)\right| \leqslant 2\|f-g\|_{C\left[0, b_{n}\right]}+\frac{x\left(b_{n}-x\right)}{2[n]_{q}}\|g\|_{C^{2}\left[0, b_{n}\right]}
$$

and hence

$$
\left|C_{n, q_{n}}(f ; x)-f(x)\right| \leqslant 2\|f-g\|_{C\left[0, b_{n}\right]}+\frac{B b_{n}}{2[n]_{q_{n}}}\|g\|_{C^{2}\left[0, b_{n}\right]} .
$$

Taking infimum over all $g \in C^{2}\left[0, b_{n}\right]$, we obtain the desired result which completes the proof.

Also recall that a function $f: \mathbb{R} \rightarrow \mathbb{R}$ is said to be uniform Lipschitz continuous of order $\gamma>0$ if there exists a constant $M>0$ such that

$$
|f(x)-f(y)| \leqslant M|x-y|^{\gamma}
$$

for any $x$ and $y$ in $\mathbb{R}$. In this case, we write $f \in \operatorname{Lip}(\gamma, \mathbb{R})$.

Theorem 9. Let $\left(q_{n}\right)$ be a sequence of real numbers such that $0<q_{n}<1$ and

$$
s t_{P_{p}}-\lim q_{n}=1 .
$$

If $f \in \operatorname{Lip}_{M}\left[0, b_{n}\right]$ and $x \in[0, B], B>0$ is a constant, then we have

$$
\left\|C_{n, q_{n}}(f)-f\right\|_{C\left[0, b_{n}\right]} \leqslant M\left\{\frac{B b_{n}}{[n]_{q_{n}}}\right\}^{\frac{\alpha}{2}} .
$$

Proof. The proof follows in a similar manner used in [12]. Therefore we omit the details here.

Theorem 10. Let $\left(q_{n}\right)$ be a sequence of real numbers such that $0<q_{n}<1$ and

$$
s t_{P_{p}}-\lim q_{n}=1 .
$$

Also let $\omega(\delta)$ is the modulus of continuity of $f$ on $[0, B]$ and $f(x)$ has continuous derivative as $f^{\prime}(x)$, then we have

$$
\left|C_{n, q_{n}}(f ; x)-f(x)\right| \leqslant M \sqrt{\frac{b_{n}}{[n]_{q_{n}}}} \omega\left(f, \sqrt{\frac{b_{n}}{[n]_{q_{n}}}}\right) .
$$




\section{Conclusions}

In this section, we provide an example such that the sequence $\left(q_{n}\right)$ satisfies neither the conditions of the results obtained in [12] nor the conditions of the results obtained in [23].

Example 1. Let the sequences $\left(p_{n}\right)$ and $\left(q_{n}\right)$ defined as follows:

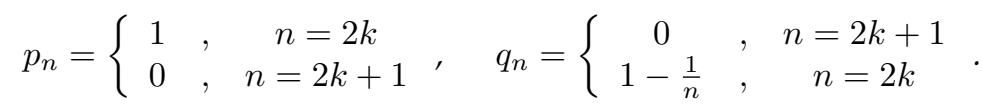

It is easy to see that the method $P_{p}$ is regular and one can easily see that

$$
\delta_{P_{p}}\left(E_{\varepsilon}\right)=0
$$

where $E_{\varepsilon}=\left\{n \in \mathbb{N}_{0}:\left|q_{n}-1\right| \geqslant \varepsilon\right\}$ holds for every $\varepsilon>0$. That is $s_{P_{p}}-\lim q_{n}=1$. Notice that $\left(q_{n}\right)$ is not convergent in the ordinary sense or statistically convergent.

In [26], a sequence of positive linear operators has been presented which satisfies neither the conditions of Theorem 1 of [11] nor the conditions of the classical Korovkin theorem (Theorem 4 of [11]) but it satisfies the conditions of Theorem 5 of [11].

Here it is remarkable to mention that our results cannot be deduced from the results in [23] since $\left(q_{n}\right)$ is not convergent or statistically convergent.

\section{Acknowledgment.}

We thank the reviewer for their insightful comments and suggestions that helped us improve the paper.

\section{Funding}

There is no funding for this work.

\section{Availability of data and materials}

Not applicable.

\section{Competing interests}

The authors declare that they have no competing interests.

\section{Author's contributions}

All authors contributed equally to the writing of this paper. All authors read and approved the final manuscript.

\section{References}

[1] Acar İşler, N.: Bernstein operator approach for solving linear differential equations. Mathematical Sciences and Applications E-Notes. 9(1), 28-35 (2021).

[2] Altomare, F., Campiti, M.: Korovkin type approximation theory and its applications. De Gruyter Studies in Mathematics. 17, Walter de Gruyter\&Co. Berlin (1994).

[3] Atlıhan, Ö.G., Orhan, C.: Summation process of positive linear operators. Computers and Mathematics with Applications. 56, 1188-1195 (2008).

[4] Atlıhan, Ö.G., Ünver, M., Duman, O.: Korovkin theorems on weighted spaces: revisited. Periodica Mathematica Hungarica. 75, 201-209 (2017).

[5] Bernstein, S.N.: Demonstration du theoreme de Weierstrass fondee. Communications of the Kharkov Mathematical Society. 13, 1-2 (1912).

[6] Boos, J.: Classical and Modern Methods in Summability. Oxford University Press. Oxford (2000). 
[7] Chlodowsky, I.: Sur le developpement des fonctions definies dans un intervalle infini en series de polynomes de M. S. Bernstein. Compositio Mathematica. 4, 380-393 (1937).

[8] Duman, O., Orhan, C.: Statistical approximation by positive linear operators. Studia Mathematica. 161, 187-197 (2004).

[9] Fast, H.: Sur la convergence statistique. Colloquium Mathematicum. 2(3/4), 241-244 (1951).

[10] Fridy, J. A.: On statistical convergence. Analysis 5, 301-313 (1985).

[11] Gadjiev,A.D., Orhan, C.: Some approximation theorems via statistical convergence. Rocky Mountain Journal of Mathematics. 32, 129-137 (2002).

[12] Karsli, H., Gupta, V.: Some approximation properties of q-Chlodowsky operators. Applied Mathematics and Computation. 195, 220-229 (2008).

[13] Korovkin, P.P.: On convergence of linear positive operators in the space of continuous functions. Doklady Akademii nauk SSSR. 90, 961-964 (1953).

[14] Kratz, W., Stadtmüller, U.: Tauberian theorems for $J_{p}$-summability. Journal of Mathematical Analysis and Applications. 139, 362-371 (1989).

[15] Lorentz, G. G.: Bernstein Polynomials. University of Toronto Press. Toronto (1953).

[16] Lupaş, A.: A q-analogue of the Bernstein operator, seminar on numerical and statistical calculus. University of Cluj-Napoc. 9, 85-92 (1987).

[17] Oruc, H., Tuncer, N.: On the convergence and iterates of q-Bernstein polynomials. Journal of Approximation Theory. 117, 301-313 (2002).

[18] Ostrovska, S:, On the improvement analytic properties under the limit q-Bernstein operator. Journal of Approximation Theory. 138, 37-53 (2006).

[19] Ostrovska, S.: q-Bernstein polynomials and their iterates. Journal of Approximation Theory. 123, 232-255 (2003).

[20] Özgüç, İ., Taş, E.: A Korovkin-type approximation theorem and power series method. Results in Mathematics. 69(3) (2016).

[21] Phillips, G.M.: Bernstein polynomials based on the q-integers. Annals of Numerical Mathematics. 4, 511-518 (1997).

[22] Salat, T.: On statistically convergent sequences of real numbers. Mathematica Slovaca. 30(2), 139-150 (1980).

[23] Taş, E., Orhan, C., Yurdakadim, T.: The Stancu-Chlodowsky operators based on q-Calculus. In: Proceedings of the 11th International Conference of Numerical Analysis and Applied Mathematics (ICNAAM), Sept 21-27 2013, Rhodes Island, GREECE. AIP Conference Proceeding. 1558, 1152-1155 (2013).

[24] Taş, E., Yurdakadim, T., Atlihan, Ö.G..: Korovkin type approximation theorems in weighted spaces via power series method. Operators and Matrices. 12, 529-535 (2018).

[25] Uysal, G., Y1lmaz, B.: On convergence of partial derivatives of multidimensional convolution operators. Mathematical Sciences and Applications E-Notes. 9(1), 9-21 (2021).

[26] Ünver, M., Orhan, C.: Statistical convergence with respect to power series methods and applications to approximation theory. Numerical Functional Analysis and Optimization. 40(5), 535-547 (2019).

[27] Wang, H.: Voronovskaja-type formulas and saturation of convergence for $q$-Bernstein polynomials for $0<q<1$. Journal of Approximation Theory. 145, 182-195 (2007). 


\section{Affiliations}

HALIME TAŞER

AdDRESS: Bilecik Şeyh Edebali University, Bilecik, Turkey.

E-MAIL: halime.taser@bilecik.edu.tr

ORCID ID: 0000-0003-2338-9242

TUĞBA YURDAKADIM

AdDRESS: Bilecik Şeyh Edebali University, Department of Mathematics, Bilecik 11230, Turkey.

E-MAIL: tugbayurdakadim@hotmail.com

ORCID ID: 0000-0003-2522-6092 